\title{
¿ES POSIBLE BAJAR LAS TASAS DE INTERÉS?
}

\author{
MARIO ZAMBRANO BERENDSOHN \\ - Profesor de Administración de Riesgos \\ Carrera de Administración y Finanzas \\ Facultad de Negocios, UPC
}

\begin{abstract}
Un tema que no solo se mencionó en la reciente campaña electoral, sino que forma parte de la habitual discusión en medios es la posibilidad de bajar las tasas de interés de los créditos, especialmente para la pequeña empresa, y de las tarjetas de créditos. A continuación, analizaremos de forma simplificada los factores que conforman la tasa de interés y qué medidas o espacios estarían disponibles para su disminución, empezando, primeramente, con un análisis de los factores de costo como i) la remuneración del fondeo, ii) el encaje legal, iii) las provisiones regulatorias; y iv) los gastos de administración.
\end{abstract}

En cuanto al primer punto, el sistema financiero tiene un amplio espectro de alternativas de fondeo o costos financieros: baratos desde las cuentas corrientes y de ahorro para bancos de excelente rating así como las emisiones en el mercado monetario y en el de capitales, hasta más onerosos como, por ejemplo, tomar prestado de otros bancos o captar ahorros siendo una empresa financiera con un rating mediano o de mayor riesgo. En lo que va de 2016, se observa que las empresas del sistema financiero están ofreciendo tasas de captación más altas en soles, lo que denotaría una escasez relativa de dicha moneda. En ese sentido, cabe preguntarse la razón de la menor disposición de fondos en soles en esta coyuntura; y por ende, si las condiciones de liquidez podrían inhibir el potencial traspaso. De otro lado, desde el punto de vista de las expectativas sobre el costo de fondeo en soles, un elemento para el análisis lo constituye el manejo de la tasa de referencia por parte de la máxima autoridad monetaria, que en los últimos meses ha tenido desplazamientos con la finalidad de conducir la dinámica de la inflación.

\section{EN LO QUE VA DE 2016, SE OBSERVA QUE LAS EMPRESAS DEL SISTEMA FINANCIERO ESTÁN OFRECIENDO TASAS DE CAPTACIÓN MÁS ALTAS EN SOLES, LO QUE DENOTARÍA UNA ESCASEZ RELATIVA DE DICHA MONEDA.}

De otro lado pero vinculado a la liquidez en la economía, cabe preguntarse si la reciente aprobación del retiro del 95.5\% de los fondos de jubilación, significará un traspaso de la liquidez desde el sistema privado de pensiones al sistema bancario en general y a otras industrias financieras, como los fondos mutuos por ejemplo, impactando, de persistir, en la "guerra" de tasas pasivas.

En cuanto al encaje legal, que responde a objetivos de la política monetaria, se plasma en que los bancos tienen que cobrar más que la tasa que pagan de fondeo solo para tener un resultado financiero cero. Ejemplo: si el banco capta 100 al 2\% tendrá que devolver 102; y producto de esa captación, podrá prestar como máximo 95, asumiéndose un encaje del $5 \%$ sobre lo que se fondeó, manteniendo 5 en caja o en cuenta corriente. Entonces ¿qué tasa debería cobrar por los 95 disponibles a prestar para ganar 102 y cubrir su costo financiero? claramente una tasa superior al $2 \%$ aproximadamente $7 \%$ en este caso.

\section{..EMPÍRICAMENTE, EN LOS ÚLTIMOS AÑOS, LA PÉRDIDA ESPERADA QUE MÁS HA SUBIDO CORRESPONDE TANTO A LA PEQUEÑA COMO A LA MEDIANA EMPRESA. ENTONCES, ACÁ ESTAMOS HABLANDO DE UN SOBRECOSTO AL TíPICO PREMIO POR RIESGO.}

El tercer factor de costo que identificamos al principio trata de las provisiones regulatorias. Respecto a este factor, hay que tener presente que la legislación financiera peruana requiere en promedio un $1 \%$ de provisión, por clasificación "normal", o gasto por cada crédito otorgado y que se asocia a la pérdida esperada al momento de otorgar un préstamo. Es decir, la tasa de provisión se añade a los demás factores para formar la tasa de interés que se cobra a los deudores. No obstante, empíricamente, en los últimos años, la pérdida esperada que más ha subido corresponde tanto a la pequeña como a la mediana empresa. Entonces, acá estamos hablando de un sobrecosto al típico premio por riesgo. En otras palabras, las empresas financieras podrían estar constituyendo una tasa de provisión interna mayor a la regulatoria debido a la mayor pérdida esperada.

En cuanto al factor de gastos administrativos y su eficiencia, finalmente, es un elemento que como cualquier negocio se incluye en el precio, en este caso en la tasa de interés. Siendo para el negocio de créditos un tema clave el alcanzar volumen, a mayor escala de operaciones la importancia de estos gastos se diluye, es decir, la eficiencia permitiría que la incidencia de este factor pudiera disminuir y por ende la tasa. No obstante, son pocas empresas financieras en el Perú las que tienen un volumen de negocios elevado (i.e., masa de clientes y/o operaciones a gran escala), y por ende ratios de gastos administrativos sobre activos productivos relativamente bajos. En la Tabla 1, se aprecia que en términos de márgenes operativos, los gastos administrativos representan aproximadamente el 50\%. 


\section{Tabla 1: Ratios de representación de los gastos} administrativos

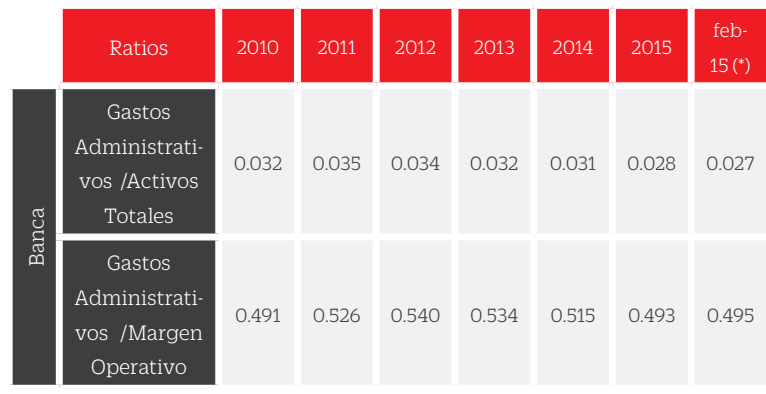

${ }^{*}$ ) Gastos administrativos y margen operativo anualizados

Fuente: SBS

La sumatoria de estos cuatro factores forma la base de las tasas de interés activas, de la que se parte sólo para cubrir costos. A esta tasa base hay que sumar el spread por el riesgo de crédito de cada potencial deudor. Esta prima de riesgo, también llamada "pérdida no esperada", es diferente entre los tipos de créditos y también al interior de cada tipo de crédito. Por ejemplo, el crédito corporativo puede ser de un menor riesgo que el de una tarjeta de crédito o al de una pequeña empresa; y prestarle o darle una tarjeta de crédito a una persona con poca información (i.e., antecedentes o historial crediticio), o con ingresos no permanentes, o con muchas tarjetas en su haber (i.e., potenciales sobre endeudados), sería de mayor riesgo, que a una persona con una excelente reputación y antecedentes de pago en el sistema, así como ingresos no sólo menos temporales sino en alza. Así, hay un spread que incorporar en la tasa de interés a cobrar debido a las primas de riesgo de crédito, plazo, etc. Es decir, a mayor riesgo, mayor prima, y por tanto, mayor tasa. Debe destacarse que la prima por riesgo de crédito no es un factor que se pueda disminuir por alguna regulación o decreto porque depende directamente de la capacidad y voluntad de pago del deudor, que a su vez está en función de otros factores, distintos, que no entran en el análisis de la estructura de la tasa de interés.

Por otro lado, se puede discutir el potencial impacto que otras variables tendrían en la tasa de interés, por ejemplo, el pago de las contribuciones al supervisor de banca y al fondo de seguro de depósitos; además que el sistema regulador bancario peruano tiene otras provisiones adicionales diferentes a las de la clasificación crediticia (provisiones pro cíclicas, por alineamiento, o por riesgo cambiario crediticio), aunque se puede decir que los primeros factores nombrados serían los de mayor materialidad.

Entonces, la tasa base más el spread por riesgo de crédito debería ser la tasa que podría observarse en los tarifarios individuales correspondientes de la banca; y teniendo en cuenta que cada empresa financiera puede tener distintas tasas o tarifarios pues cada banco o caja tiene diferente costo de fondeo o tiene una mayor o menor eficiencia de gastos administrativos, por ejemplo.

Las tasas de interés activas, por encima de las tasas base más el spread por riesgo, son aquellas que podrían bajarse a través de una mayor transparencia y/o con una mayor competencia en el sistema. En el primer caso, podría ganarse aún mayores niveles de información, a través de acciones de los oferentes de crédito hacia el mercado, debido a condiciones establecidas por el regulador. En el segundo caso, la reducción de tasas por mayor competencia pudiera ocurrir -en mayor grado- en los deudores ya "bancarizados" y con clasificación de riesgo "normal", aunque habría evidencia que actualmente existe ya algún grado de competencia en el negocio de tarjetas de crédito en el Perú.

La competencia podría profundizarse conforme empresas financieras, como por ejemplo las cajas municipales, decidieran incursionar en este nuevo negocio; decisión que implicará evaluar inversiones en infraestructura, otra tecnología crediticia intensiva en sistemas y modelos, y sobre todo a una elevada escala o volumen de tarjeta-habientes para que sea una actividad rentable. Actualmente las cajas tienen un modelo de negocios orientado principalmente al cliente micro; y su participación en el segmento consumo no es la principal.

Otro elemento de competencia a analizar correspondería a la industria de intermediación financiera no regulada, como es el conjunto de cooperativas, que en el Perú suman más de 165, y que no han pasado por el proceso de autorización de organización y funcionamiento de la Superintendencia de Banca y Seguros (SBS). Esta es una industria donde su modelo de negocios se enfoca principalmente en los créditos de consumo y donde cualquiera de las tres cooperativas más grandes es inclusive de mayor tamaño que el promedio de cajas municipales e inclusive que varios bancos. Es decir, es un sector con un tamaño de operaciones activas que al pasar al mercado regulado podría generar algún impacto sobre el segmento consumo, incluyendo mayor información a la central de riesgos, lo que posibilitaría una mejor gestión del riesgo de crédito.

Finalmente, de acuerdo a data pública disponible, se observa una tendencia a la baja en las tasas de interés activas en soles para la pequeña empresa y una estabilidad para el segmento de consumo; y en lo más reciente una disminución de las tasas en ambos segmentos de negocio -- ver Tabla 2.

\section{Tabla 2: Tasas activas para pequeña empresa y} consumo

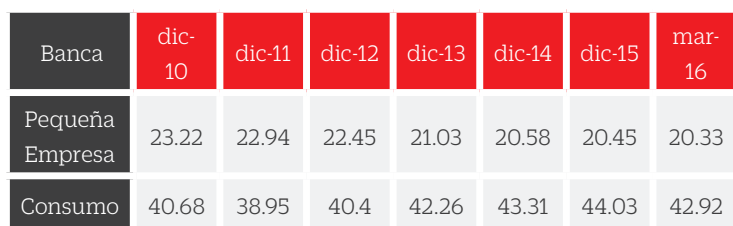

Fuente: SBS. Tasas anuales porcentuales.

En resumen, hay pocos factores que en el corto plazo se pudieran ajustar y más bien parece que son los factores estructurales, aquellos que requieren de mayor tiempo en cambiarse, en los que habría que seguir avanzando para en tendencia bajar las tasas activas; y ello incluye continuar con los esfuerzos de bancarización, transparencia y competencia. 